\title{
Review
}

\section{Progress in the Prevention and Treatment of AIDS Associated with Tuberculosis}

\author{
Wenlong He, Rui Li, Yaogang Wang \\ Tianjin Medical University, Tianjin 300010, China
}

\author{
Keywords \\ HIV and TB co-infection; epidemic; control \\ policy \\ Correspondence \\ Yaogang Wang, \\ E-mail:wyg@tmu.edu.cn \\ DOI: 10.1515/ii-2017-0129
}

\begin{abstract}
Epidemiological studies have shown that infection with the human immunodeficiency virus (HIV) is an influential risk factor for infection with Mycobacterium tuberculosis (MTb), the rapid progression of the initial infection to active tuberculosis (TB), and the reactivation of latent TB infection. MTb infection is also one of the most common opportunistic infections in people with HIV, including AIDS patients receiving anti-retroviral therapy. Given the prevalence of HIV infection, the incidence of TB infection, which had begun to decline, is facing a severe situation. HIV associated with TB exerts an immense burden on the public health-care system, especially in countries with high incidences of HIV infection. Therefore, the global policies for the prevention and control of TB should be revised. Moreover, an increased investment in TB control has to be guaranteed. The purpose of this review is to summarize the recent progress in the prevention, treatment, and control of HIV and TB co-infection.
\end{abstract}

Tuberculosis (TB), an infectious disease caused by Mycobacterium tuberculosis (MTb), is a major cause of human death. According to a report by the World Health Organization (WHO), approximately $1 / 3$ of the world's population is infected with $\mathrm{MTb}$. The majority of $\mathrm{TB}$ patients is infected with latent TB infection (LTBI). MTb can remain dormant in the macrophages of LTBI-infected individuals for a long duration. When the host's immunity decreases, dormant MTb begin to multiply and eventually develop into tuberculosis. Human immunodeficiency virus (HIV) infection destroys the immune system of patients with acquired immune deficiency syndrome (AIDS). Therefore, HIV infection is the main reason that $\mathrm{TB}$ cannot be controlled in areas with high AIDS incidence. In addition, TB is the most common opportunistic infection among HIV carriers and the leading cause of death in patients with AIDS. HIV and MTb co-infection has not only become a serious medical hazard to human health but also greatly damages society and economy, thus becoming a global public health problem. This review provides a summary of the latest progress in the global policies for the prevention and treatment of AIDS associated with TB.

\section{Prevalent status of AIDS and TB}

TB is one of the most ancient infectious human diseases and has been extant for centuries ${ }^{[1]}$. From 1990 to 2012, the total global TB cases decreased by $37 \%$ and the TB mortality rate decreased by $45 \%$. These encouraging figures seemed to indicate that by 2015, the number of new TB cases would have declined by $50 \%$ and mortality would have declined by $75 \%$ compared with those in $1990^{[2]}$. However, due to the imbalance in economic development and resource allocation, as well to extreme differences in medical and health conditions among different regions, the global control of TB remains challenging, particularly in poor regions. In 2012, 8.6 million new TB cases, including 1.3 million deaths from TB, were reported in the world. Among these cases, $80 \%$ occurred in 22 known high-risk countries. The number of new cases in China ranks second in the world and is next only to India ${ }^{[3]}$. TB epidemics, which have been controlled effectively for a long time, suddenly resurged with the prevalence of HIV infection. In 2012, 13\% of newly reported TB infected patients were co-infected with HIV. In Africa, $4 / 5$ of TB patients were co-infected with HIV. AIDS cases 
associated with TB accounted for approximately $25 \%$ of TB death cases. In Asia, this figure was 40\% ${ }^{[4]}$. Epidemiological studies have shown that as two major public health issues in the world, AIDS and TB promote each other in prevalence. Of the approximately 34 million HIV-infected people in the world, more than $1 / 3$ are co-infected with TB (including latent infection). In addition, the incidence of $\mathrm{HIV}$ and $\mathrm{TB}$ co-infection is 30 times of that TB infection only. TB is also one of the most common opportunistic infections in HIVinfected people, including AIDS patients receiving antiretroviral therapy. Statistics show that in China, the infection rate of TB is approximately $7 \%$ in HIV-infected people and AIDS patients. The infection rate of TB among AIDS patients is approximately $20 \%{ }^{[5]}$. TB is also the primary cause of death among AIDS patients. Based on the statistics reported by the WHO, 1 out of 5 AIDS patients will eventually die from TB. More seriously, HIV-infected individuals face the risk of infection by multidrug-resistant tuberculosis (MDRTB) and extensively drug-resistant tuberculosis (XDRTB) ${ }^{[6]}$. A clinical study in South Africa showed that the fatality rates of AIDS patients with MDR-TB or XDR-TB coinfection are $98 \%$ and $100 \%$, respectively ${ }^{[7]}$.

\section{Prevention strategy of AIDS associated with tuberculosisn}

HIV and TB not only promote each other in prevalence and interact in pathogenesis. Although the specific mechanisms that underlie the co-infection of HIV with TB have not yet been fully elucidated, HIV and MTb infections can weaken the body's immune system. HIV infection is the most important risk factor for the ultimate development of $\mathrm{MTb}$ infection into tuberculosis. HIV infection generally decreases immune system function. In this situation, latent $\mathrm{MTb}$ infection will develop into active tuberculosis. HIV infection associated with TB is not only the simple sum of the process and effects of independent infection with the two pathogens: synergism between both pathogens accelerates disease progression and promotes epidemics. Individuals infected with HIV and AIDS (HIV/AIDS patients) are highly susceptible to TB. The prevention of TB in HIV/AIDS patients can decrease the fatality rate of the two diseases, as well as control their prevalence. At present, different strategies are adopted in different countries for the prevention and control of HIV infection associated with TB, as follows:

\section{Ukraine}

Early TB screening has been implemented among the mostat-risk population (MARP) since April, $2013^{[8]}$. Thirty-nine non-governmental organizations (NGOs) cooperate with medical institutions around Ukraine to implement early testing for TB infection among MARP under the support of the TB Alliance. MARP includes people who inject drugs (PWID) or injecting drug user (IDU), commercial sex workers (CSW), and men who have sex with men (MSM). After training in TB testing and prevention methods, NGO community workers use questionnaires to first screen for TB symptoms among the MARP population. Then, the workers collect sputum specimens on site for TB testing at the local medical institution. The test result is used as the basis for determining whether an infection control program should be initiated. The results of this method have shown that the detection rate of TB infection among the MARP population is far higher than that among the general population in medical institutions. Therefore, the active detection of TB among the MARP population can decrease the propagation and fatality rates of TB in the MARP population.

\section{Indonesia}

The 2010-2014 Indonesia National TB-HIV Action Plan ${ }^{[9]}$ for active TB screening integrates active TB detection into health services for the key population (KP), which includes PWID and prisoners, and provides HIV detection tests to all TB patients. HIV/MTb co-infection has complicated clinical manifestations, a low positive rate in sputum, and a low positive rate in the tuberculin test. For TB screening among the KP population, the Indonesia National Guideline has adopted Xpert MTB/Rif ('Xpert', Cepheid, Sunnyvale, California, USA) a TB test kit applicable for GeneXpert device. This kit can be used to detect the resistance of Mtb to rifampin. Among PWID who seek methadone for maintenance and needle syringe exchange program services, more than $20 \%$ (more than $90 \%$ at most) have obvious TB symptoms and $100 \%$ of these TB patients have HIV. The Indonesian government provides directly observed therapy (DOT) and anti-retroviral therapy (ART) to these patients.

\section{South Africa}

Xpert MTB/Rif is used to diagnose rifampicin-resistant TB (RR-TB) to facilitate the immediate initiation of therapy [10]. A total of 5,100 TB cases are annually reported in Khayelitsha, South Africa, which has a total population of 
0.4 million. Among these cases, the positive rate of HIV is $75 \%$ with approximately 200 RR-TB cases. With the use of Xpert, the median time from diagnosis to initial treatment has been reduced to 7 days, and more than $90 \%$ of HIV cases associated with RR-TB can be treated in time. With Decentralisation RR-TB treatment, the treatment period has been shortened to 1-2.51 months, greatly decreasing the fatality rate of HIV/RR-TB co-infected persons.

\section{China}

China is a country with a high incidence of tuberculosis. The AIDS epidemic in China contributes to the increasing incidence of tuberculosis. In addition, HIV infection associated with MTb has increased in China. An epidemiological study has shown that the TB infection rate of AIDS patients is $29.1 \%$, and that TB has become the main reason for the hospitalization and death of AIDS patients in China. These results indicated that TB seriously affects the prognosis and quality of life of AIDS patients ${ }^{[5,14-15]}$. With support from major national science and technology projects, Chinese researchers have conducted preliminary studies on the epidemiological characteristics, related influential factors, diagnosis, and treatment of HIV infection associated with $\mathrm{MTb}$ and latent $\mathrm{TB}$ infection. Chinese researchers have used biomarkers, such as the dynamic changes in serum cytokine levels, to diagnose TB infection ${ }^{[11-13]}$.

Moreover, Chinese scientists have investigated new technology for the diagnosis of HIV infection associated with MTb. Researchers have evaluated the sensitivity, specificity, and practicability of -SPOT.B, a commercial $\gamma$-Interferon release assay, in the detection of the tubercle bacillus in HIV patients with MTb. Preliminary results showed that the sensitivity of T-SPOT.B is insufficient to support TB screening in HIV-infected individuals. Given its high specificity, however, it can be used as an assistant tool for TB diagnosis ${ }^{[16-17]}$. In addition to the development of new diagnostic technologies and methods for $\mathrm{TB}$, standardizing the diagnosis and treatment measures of AIDS associated with tuberculosis is also key to decreasing the incidence and fatality rate of AIDS associated with TB. During the eleventh 5 -year plan, researchers in China explored the diagnostic and therapeutic strategies for AIDS to establish the suitable standard diagnosis and treatment strategy for Chinese AIDS patients. The developed standardized diagnosis and treatment programs should be further popularized, applied, and further optimized to improve antiviral efficacy and decrease the incidence and fatality rate of AIDS and HIV coinfection.

Preventative measures taken by countries around the world in response to HIV infection associated with $\mathrm{MTb}$ include: 1) The promotion of NGOs that actively participate in the early detection of HIV/MTb infection, and that enhance the connection among itself, governmental departments, and public health organizations to provide treatment to infected patients. 2) The elimination of discrimination against and the integration of high-risk groups susceptible to HIV. The extension of TB testing services, DOT services, and ART treatment to high-risk groups. 3) The adoption of more advanced TB detection methods to improve the detection rate of MDR-TB and shorten the period between diagnosis to treatment.

\section{Treatment of AIDS complicated with tuberculosis}

Since 2010, the WHO has recommended that all HIV patients co-infected with TB should receive ART 8 weeks within starting anti-TB treatment regardless of CD4+T lymphocyte (CD4 cells) count. Patients with severely suppressed immune systems $(\mathrm{CD} 4$ cell count $<50 / \mu \mathrm{L})$ should begin ART 2 weeks within starting TB treatment ${ }^{[18]}$. At present, the standard first-line TB treatment is continued for 6 months. After intensive treatment, isoniazid with rifampicin, pyrazinamide, and ethambutol are continued for 2 months. Isoniazid and rifampicin are then used as maintenance medication for 4 months ${ }^{[19]}$. According to the latest guideline for highly active anti-retroviral therapy (HAART) ${ }^{[20]}$, HIV/AIDS patients receiving anti-TB therapy should be treated with efavirenz (EFV) as a non-nucleoside reverse transcriptase inhibitor (NNRTIs). Rifabutin, a protease inhibitor (PI), should be considered as the secondline drug for AIDS. Given that HIV and MTb promote and interact with each other, both diseases must be treated simultaneously. However, the simultaneous application of anti-TB and anti-HIV drugs remains problematic ${ }^{[21]}$ because of the following reasons: 1) The adverse reactions to the two kinds of drugs are cumulative, synergistic, and are especially toxic in the liver and gastrointestinal tract. Drug administration must sometimes be stopped during treatment. 2) Immune reconstitution inflammatory syndrome (IRIS) is a risk and side effect of treatment. 3) During drug metabolism, rifampicin will interact with 
NNRTIs and PIs, thus affecting the effectiveness of ART treatment. In addition, rifampicin upregulates the expression levles of Cytochrome P450 (CYP450) and CYP3A protein to promote the metabolism of ART drugs and decrease their plasma levels. Rifampicin will also induce the expression of other CYP isozymes. Moreover, at stage II, rifampicin will induce the expression of drug metabolic enzymes, such as UDP-glucuronyltransferases and dulfotransferases. Rifampicin upregulates the expression of some transport proteins, including $\mathrm{P}$-glycoprotein and multiple drug resistance protein 2 (MRP2). 2) Unlike Rifampicin, isoniazid inhibits some CYP450 family members (CYP2C9, CYP2C19, and CYP2E1). 3) Other anti-TB first-line drugs, including pyrazinamide and ethambutol, exert no known regulatory effects on the above-mentioned drug metabolic enzymes.

Rifampicin interacts with HAART drugs as follows:1) Interaction with NNRTIs. Efavirenz is mainly degraded by CYP2B6, which is expressed in the liver. Therefore, rifampicin decreases the plasma concentration of Efavirenz. Notably, a gene polymorphism of CYP2B6 (CY P2B6, 516G-T) blocks the metabolism of Efavirenz to maintain high plasma concentrations of Efavirenz. The weight and ethnicity of patients also influence the interaction of NNRTIs with HAART drugs. Therefore, the plasma concentration of the drugs should be carefully monitored. 2) Interaction with PIs. Almost all HIV PIs recommended by the WHO are substrates for CYP3A4. Therefore, interaction with rifampicin is inevitable. Given that the metabolic products of PIs are transported by P-glycoprotein, rifampicin can decrease plasma PI concentrations by $80 \%-95 \%$. Thus, when PIs are simultaneously used with rifampicin, the effective concentration and toxicity of PIs should be fully considered. 3) Interaction with NRTIs. NRTIs do not easily interact with rifampicin. Therefore, NRTIs can be used with rifampicin.

\section{Challenges and Prospects}

HIV infection hinders the control and treatment of TB. Although this review has shown the interaction between HIV infection and TB and the mutual influencing mechanism between the two diseases, these mechanisms should be further studied through additional scientific experiments and advanced research studies. After many years of practice, clinicians have reached a preliminary consensus on the prevention and treatment of HIV/AIDS patients co-infected with TB. Preliminary studies have shown that the detection rate of TB among the MARP population is far higher than that among the general population. Therefore, NGOs have developed community outreach programs for TB detection in MARP. To prevent and treat HIV/MTb co-infection, medical institutions have cooperated with NGOs to provide early TB treatment to susceptible populations. However, the medical health system has widespread discrimination against MARP groups, especially PWID. This discrimination hinders MARP groups from receiving a wide range of medical and health services. In addition, the health system has an insufficient awareness of the early diagnosis and treatment of TB. Thus, the service for the early diagnosis and treatment of TB remains insufficient. Moreover, MARP individuals are less concerned about their health because of their habits, customs, and low social and economic status.

HIV/AIDS patients co-infected with MTb still face many problems in clinical treatment, such as the selection of the best time for treatment with anti-TB and anti-HIV drugs, the optimization of medication regimens, and the elimination mechanism of adverse reactions. A total of $8 \%$ $-36 \%$ of HIV/AIDS patients co-infected with MTb suffer from IRIS after anti-TB and ART. IRIS aggravates clinical symptoms and endangers the lives of patients when serious. The clinical diagnosis of IRIS continues to face numerous challenges. For example, MDR-TB hinders the treatment of IRIS. In addition, IRIS is associated with bacterial or fungal infections, with tumors, and with adverse reactions to drug treatment. At present, studies on IRIS still focus on the optimization of diagnosis and clinical treatment strategies. Its pathogenesis and immunological mechanisms remain unclear. Some international cross-sectional studies and follow-up observations have shown that compared with a non-IRIS group: 1) The frequency of Type I helper T-cell (Th1) with TB antigen specificity in the peripheral blood of TB-IRIS patients is significantly higher ${ }^{[22]}$. 2) Prior to HAART treatment (baseline), the frequency of $\gamma \delta+$ T-cells expressing killer inhibitory receptor (KIR) in the peripheral blood of TB-IRIS patients significantly decreases ${ }^{[23]}$. 3) Although the frequency and quantity of regulatory T-cells in the peripheral blood of TB-IRIS patients are not significantly changed ${ }^{[24]}$, the inhibition of T-cell activation is significantly weakened ${ }^{[25]}$. Latest studies on a South African cohort have found that the frequency and activation of peripheral blood CD14++CD16 is an independent predictive factor for 
the occurrence of TB-IRIS ${ }^{[26]}$. Moreover, cohort studies in Malaysia and India have shown that serum IL-18 levels can be used to predict the occurrence of TB-IRIS ${ }^{[27]}$.

These studies have some shortcomings or unanswered questions for further study: 1) The number of queues should be increased. 2) Immune cells are derived from peripheral blood, not the infected site or lesion. 3) The molecular mechanism that underlies the loss of T-reg function in TB-IRIS patients remains unknown. 4) The molecular mechanism for the rapid recovery of Th1 cells in the body of TB-IRIS patients and its phenotype and function should be determined. 5) The role of other immune cells and cytokines in the pathogenesis of TB-IRIS.

To summarize, decreasing the incidence and fatality rate of TB among AIDS patients require the identification of the recurrence rate and reinfection rate of AIDS patients co-infected with TB after being treatment. In addition, the factors that affect the prognosis and curative effect of AIDS associated with TB should be analyzed. The principles of immune reconstitution and of IRIS pathogenesis in AIDS patients co-infected with TB should be identified. The incidence of drug-resistant tuberculosis should be observed. These topics should receive more attention for the development of preventative measures against AIDS and TB co-infection.

\section{Declarations}

\section{Acknowledgements}

No.

\section{Competing interests}

The authors declare that they have no competing interest.

\section{Authors' contributions}

WL He and R Li made the literature analysis and wrote, discussed and revised the manuscript of this review. YG Wang critically analyzed and corrected the manuscript. All authors read and approved the final manuscript.

\section{References}

1 Hershkovitz I, Donoghue HD, Minnikin DE, et al. Detection and molecular characterization of 9,000-year-old Mycobacterium tuberculosis from a Neolithic settlement in the Eastern Mediterranean. PLoS One,2008,3(10):e3426.
2 World Health Organization. Global strategy and targets for tuberculosis prevention, care and control after 2015. 2013.

3 World Health Organization.Global Tuberculosis Control:Surveillance, Planning, Financing. 2008.

4 World Health Organization.Global Tuberculosis Report 2013. 2013.

5 Gao L, Zhou F, Li X, et al. HIV/TB co-infection in mainland China: a meta-analysis. PLoS One, 2010, 5(5): e10736.

6 Editorial.Co-infection:new battlegrounds in HIV/AIDS. Lancet Infect Dis, 2013, 13(7): 559 .

7 Singh JA, Upshur R, Padayatchi N. XDR-TB in South Africa: no time for denial or complacency. PLoS Med, 2007, 4(1): e50.

8 Islam Z. Presentation at 2014 AIDS International Conference. 2014.

9 Nadia S, Mustikawati DE. Presentation at 2014AIDS International Conference. 2014.

10 Cox H, Hughes J, Moyo S, et al. Presentation at 2014AIDS International Conference. 2014.

11 Diallo MA, Chen X, Zhen Y, et al. Epidemiological features and related influencing factors of HIV associated with tuberculosis infection. Journal of Chinese Physician, 2011, 13(1): 1-4.

12 Wang Y, Qi Y, Wan R, et al. Comparison of Mycobacterium tuberculosis detection techniques and drug resistance phenotypes in patients with HIV/AIDS complicated with tuberculosis. Chinese Journal of clinicians, 2012, 4(6): 2115-2119.

13 Fu Y, Zhang Z, Liu F, et al. Serum cytokine levels in patients with HIV/ TB co- infection in China. J China Med Univ, 2014, 43(3): 196-200.

14 Shen Y. Anti-TB treatment of AIDS patients associated with TB. SH PHARMA, 2009, 30(1): 8-10.

15 Zhu C. Prevalence and drug resistance of tuberculosis. SH PHARMA 2009, 30(1): 11-13.

16 Cai R, Chen J, Guan L, et al. Relationship between TSPOT.TB responses and numbers of circulating CD+ 4 T-cells inHIV infected patients with active tuberculosis. Biosci Trends, 2014, 8(3): 163-168.

17 Chen J, Sun J, Zhang R, et al. T-SPOT.TB in the diagnosis of active tuberculosis among HIV-infected patients with advanced immunodeficiency. AIDS Res Hum Retroviruses, 2011, 27(3): 289-294.

18 World Health Organization. WHO policy on collaborative TB/HIV activities: Guidelines for national programmers and other stakeholders. 2012.

19 World Health Organization. Treatment of tuberculosis: guideline-4th ed. 2009.

20 World Health Organization. Antiretroviral therapy for HIV infection in adults and adolescents: Recommendations for a public health approach: 2010 revision. 2010.

21 Semvua HH, Kibiki GS, Kisanga ER, et al. Pharmacological interactions between rifampicin and antiretroviral drugs: challenges and research priorities for resource-limited settings. Ther Drug Monit, 2015, 37(1): 22-32. 
22 Meintjes G, Wilkinson KA, Rangaka MX, et al. Type 1helper T cells and FoxP3-positive T cells in HIV-Tuberculosis-associated immune reconstitution inflammatory syndrome. Am J Respir Crit Care Med, 2008, 178: 1083-1089.

23 Bourgarit A, Carcelain G, Samri A, et al. Tuberculosis-associated immune restoration syndrome in HIV-1-infected patients involves tuberculinspecific CD4Th1cells and KIR-negative gammadelta T cells. J Immunol, 2009, 183(6): 3915-3923.

24 Zaidi I, Peterson K, Jeffries D, et al. Immune reconstitution inflammatory syndrome and the influence of $\mathrm{T}$ regulatory cells: a cohort study in The Gambia. PLoS One, 2012, 7(6): e39213.

25 Seddiki N1, Sasson SC, Santner-Nanan B, et al. Proliferation of weakly suppressive regulatory $\mathrm{CD}+4 \mathrm{~T}$ cells is associated with over-active $\mathrm{CD}+$ $4 \mathrm{~T}$-cell responses in HIV-positive patients with mycobacterial immune restoration disease. Eur J Immunol, 2009, 39(2): 391-403.

26 Andrade BB, Singh A, Narendran G, et al. Mycobacterial antigen driven activation of CD14++CD16-monocytes is a predictor of tuberculosisassociated immune reconstitution inflammatory syndrome. PLoS Pathog, 2014, 10(10): e1004433.

27 Tan HY, Yong YK, Andrade BB, et al. Plasma interleukin-18 levels are a biomarker of innate immune responses that predict and characterize tuberculosis-associated immune reconstitution inflammatory syndrome. AIDS, 2015, 29(4): 421-431. 